\title{
Bogijetong Decoction and Its Selected Formulation Are Involved in Alleviating Neuropathic Pain in a Rat Model of Chronic Constrictive Injury
}

\author{
Ki-Joong Kim, Hye-Jeong Ahn, Uk Namgung $(\mathbb{D}$, and Chung Sik Cho $\mathbb{D}$ \\ Department of Korean Medicine, Daejeon University, Daejeon 300-716, Republic of Korea \\ Correspondence should be addressed to Chung Sik Cho; choolo2@dju.kr
}

Received 7 September 2018; Revised 15 November 2018; Accepted 22 November 2018; Published 5 December 2018

Academic Editor: Ke Ren

Copyright (c) $2018 \mathrm{Ki}$-Joong Kim et al. This is an open access article distributed under the Creative Commons Attribution License, which permits unrestricted use, distribution, and reproduction in any medium, provided the original work is properly cited.

Bogijetong decoction (BGJTD) is a formulation that is used for the treatment of neuropathic pain caused by cancer therapy, diabetes, and peripheral nerve injury. In the previous study, we selected four herbal constituents from BGJTD, formulated new decoction $(\mathrm{BeD})$, and demonstrated its efficacy on the neuroprotection of peripheral sciatic nerve in streptozotocin-induced diabetic animals. Here, we report attenuating effects of BGJTD and BeD on neuropathic pain. Neuropathic pain was induced by ligation of the sciatic nerve to generate chronic constrictive injury (CCI). BeD was more effective than BGJTD in alleviating neuropathic pain lasting 3 - 4 weeks after CCI. In vivo administration of $\mathrm{BeD}$ did not alter the levels of brain-derived neurotrophic factor (BDNF) which were strongly induced by $\mathrm{CCI}$ in the sciatic nerve but downregulated TrkB production in the sciatic nerve. Downregulation of TrkB signals by $\mathrm{BeD}$ was confirmed in cultured DRG neurons. BGJTD was more effective in attenuating TNF- $\alpha$ production in the sciatic nerve than $\mathrm{BeD}$, whereas BeD increased IL-6 more efficiently than BGJTD. Furthermore, phopsho-Erk1/2 was increased in the sciatic nerve and dorsal root ganglia (DRG) after BeD treatment. Neurite outgrowth of primary DRG neurons prepared from rats which had undergone $\mathrm{CCI}$ for 7 days was significantly increased in $\mathrm{BeD}$-treated group of animals compared to the control and BGJTD-treated groups. Compositional comparison of $\mathrm{BeD}$ revealed that the neurite outgrowth was facilitated by the treatments of Panax ginseng and Paeonia lactiflora. Together, these data suggest that $\mathrm{BeD}$ induces unique molecular response at the injury site and may trigger retrograde signaling into the neuronal cell body to modulate pain responses.

\section{Introduction}

Neuropathy is peripheral nerve malady caused by diabetes, cancer, physical nerve damage, and mental illness such as depression, nicotine addiction, obesity, and stress. Clinically, neuropathic pain is considered as primary target for the treatment of neuropathy because this pain is very severe and persistent. Although $\mathrm{A} \delta$ and c-fibers are peripheral mediators of neuropathic pain, ascending spinal nerves and associated peripheral Schwann cells and spinal microglial cells are closely related to mediating pain transmission to the brain [1]. Chemical mediators such as fractalkine, substance P, ATP, and others that are secreted from these cells are known to be involved in the transmission of pain signals.

Neuropathic pain is chronic and persistent and lowers the quality of life. Neuropathic pain is not only controlled by pain-transmitting pathway but also affected by autonomic, endocrine, and immune influences $[1,2]$. Currently, surgical procedures that regulate spinal tracts and several drugs including antidepressants and anticonvulsants are used to alleviate the severity of pain, but the therapeutic approaches curing neuropathic pain are not available. Several lines of studies using experimental animals provide evidence that molecular factors that are produced and released from nerve fibers and glial cells are involved in pathological responsiveness of neuropathic pain. For instance, nerve growth factor (NGF) and brain-derived neurotrophic factor (BDNF) produced from Schwann cells and spinal microglia cells and astrocytes are involved in generating pain signals by activating TrkB receptors and low affinity p75 neurotrophin receptors (p75NTR) [3]. Fractalkine is produced from sensory neurons and induces chemotactic responses of microglial cells and consequent inflammatory responses [4]. Moreover, inflammatory cytokines such as leukocyte-inducing factor 
(LIF), IL-1 $\beta$, TNF- $\alpha$, and NF-kB are produced from peripheral and spinal glial cells and contribute to pain signaling. Tetracycline as a regulator of inflammatory cytokines and nitric oxide, propentofylline regulating reactive astrocytes, and AV411 that induces the production of anti-inflammatory cytokine IL-10 [5-7] are known to regulate pain, but their efficacy is highly variable thereby accompanied with some side effects.

BGJTD is herbal formulation composed of 18 different herbal components. Based on traditional Korean medical theory, BGJTD was developed and used for the treatment of neuropathic pain accompanied as a sequela of cancer and diabetes $[8,9]$. In order to understand biological basis of the action of BGJTD, previously we examined the effects of BGJTD on axonal regeneration after peripheral nerve injury and identified molecular factors associated with its efficacy on nerve regeneration [10]. To further characterize active herbal components among 18 different herbal components, we classified BGJTD into four subgroups BGJTD to BGJTD $_{\mathrm{d}}$ of herbal formulation based on traditional medicinal theory and constituted a new decoction $\mathrm{BGJTD}_{\mathrm{e}}$, shortly $\mathrm{BeD}$, which showed comparable efficacy to BGJTD in inducing responsiveness of injured axons [11]. While these studies strongly suggest that $\mathrm{BGJTD}$ and $\mathrm{BeD}$ are involved in regulating neuropathic pain in the injured nerves and diabetic neuropathy, its direct effect on mechanically triggered neuropathic pain remains to be studied. Here in the present study, we explored the regulatory effects of $\mathrm{BGJTD}$ and $\mathrm{BeD}$ on neuropathic pain using an animal model of chronic constrictive injury (CCI) in which the peripheral sciatic nerve was ligated. Our data provide evidence that $\mathrm{BeD}$ alleviates neuropathic pain and is involved in regulating BDNF-TrkB and IL-6 signaling pathways in the injured neurons. Furthermore, compositional analysis of $\mathrm{BeD}$ on neurite outgrowth highlights the significance of specific herbal constituents of $\mathrm{BeD}$ in regulating neuropathic pain.

\section{Materials and Methods}

2.1. Herbal Drug Extraction. BGJTD is a mixture composed of 18 different herbal drugs: Astragalus membranaceus, Panax ginseng, Angelica gigas, Rehmannia glutinosa, Cnidium officinale, Paeonia lactiflora, Salvia miltiorrhiza, Prunus persica, Carthamus tinctorius, Spatholobus suberectus, Epimedium koreanum, Lumbricidae Whole Jiangxi, Pueraria thunbergiana, Pteridium aquilinum, Albizia julibrissin, Uncaria rhynchophylla, Chaenomeles sinensis, and Crassostrea gigas [11]. We classified these constituents into 4 subgroups based on traditional medical theory, examined their effects on regenerative responses in cultured neurons, and constructed a new formula BeD composed of Panax ginseng (PG; Ginseng Radix), Crassostrea gigas (CG), Angelica gigas (AG), and Paeonia lactiflora (PL). Preparations and administration of BGJTD and BeD were performed essentially as described in our previous studies $[10,11]$.

2.2. Animals. Sprague-Dawley rats (male, 200-250 g) and mice (albino ICR; 20-25 g) were purchased from Samtako (Seoul, Korea) and adjusted in animal rooms for 1 week before the use of experiment. Animal rooms were maintained at $22^{\circ} \mathrm{C}, 60 \%$ humidity, and a $12 \mathrm{~h}$ light and $12 \mathrm{~h}$ dark cycle. Animals were allowed to eat commercial chow (Samyang Co., Korea) and drink water ad libitum. Animal care and all experimental procedures were in accordance with the NIH guide for the care and use of laboratory animals and approved by the Committee on Use of Live Animals for Teaching and Research at Daejeon University (Daejeon, Korea).

2.3. Sciatic Nerve Surgeries. Rats and mice were anesthetized with ketamine $(80 \mathrm{mg} / \mathrm{kg})$ and xylazine $(5 \mathrm{mg} / \mathrm{kg})$. The sciatic nerve on the left leg was exposed from the middle thigh between the gluteus maximus muscle and biceps femoris muscle. For crush injury (CI), the exposed nerve was held tight with a pair of forceps for $30 \mathrm{~s}$. For CCI in rats, the sciatic nerve exposed at the same area was penetrated with a sharp needle and a portion of $1 / 2$ to $2 / 3$ of the nerve was ligated tight with 5-0 and 7-0 nylon or silk threads (Ethicon, Somerville, NJ, USA). For CCI in mice, the sciatic nerve was exposed and ligation was performed similarly by using 7-0 nylon thread. Sham surgery was given on the area of the sciatic nerve by skin incision to expose the nerve and suture.

2.4. Western Blot Analysis. About $1 \mathrm{~cm}$ length of sciatic nerve and DRG at lumbar levels 4 and 5 were dissected and used to extract protein. Protein extraction, SDS-polyacryamide gel electrophoresis, blot transfer, and primary and secondary antibody reactions were performed as described previously [11]. Fifteen micrograms of protein were loaded to each lane of the gel to resolve protein. Primary antibodies used in the present study were anti-BDNF (1:1000, mouse, monoclonal, Abcam, Cambridge, MA, USA), anti-TrkB (1:800, Rabbit, polyclonal, Abcam), anti-GAP-43 (1:800, Rabbit, polyclonal, Abcam), anti-phopsho-Erk1/2 (1:1000, Rabbit, polyclonal, Cell Signaling, Danvers, MA), anti-IL-6 (1:800, mouse, monoclonal, Abcam), anti-TNF- $\alpha$ (1:800, Rabbit, polyclonal, Abcam), and anti-Actin (1:20000, mouse, Sigma) antibodies, and secondary antibodies were goat anti-mouse and goat anti-rabbit horseradish peroxidase- (HRP-) conjugated antibodies (1:1000, Cell Signaling). Quantification of protein band intensity was performed by using the i-Solution software (Image \& Microscope Technology, Daejeon, Korea).

2.5. Immunofluorescence Staining. Sciatic nerve was dissected and embedded in mounting medium at $-20^{\circ} \mathrm{C}$. Longitudinal nerve sections $(20 \mu \mathrm{m})$ were cut by using cryostat (Leica, Wetzlar, Germany) and thaw-mounted on gelatin-coated slides. Pretreament, blocking, and reactions with primary and secondary antibodies were performed as described previously [12]. Primary antibodies used in this study were aintiNF-200 antibody (1:800, monoclonal, Santa Cruz Biotech, Dallas, TX, USA), anti-NF-200 antibody (1:800, polyclonal, Santa Cruz Biotech), anti-BDNF antibody (1:400, polyclonal, Abcam), anti-TrkB antibody (1:400, monoclonal, Abcam), and anti-phospho-Erk1/2 antibody (1:400, polyclonal, Cell Signaling), and secondary antibodies were fluorescein-goat anti-mouse (1:400, Molecular Probes, Eugene, OR, USA) or rhodamine-goat anti-rabbit (1:400, Invitrogen, Carlsbad, NM, USA) secondary antibodies. Fluorescence images were 
observed by using fluorescence microscope (Nikon, Kona, Japan), captured, and analyzed by the Adobe Photoshop program.

2.6. Von Frey Tests. Behavioral measurement for pain response was performed by von Frey test. The animal was adjusted for $15 \mathrm{~min}$ by placing it on the platform of von Frey equipment. The foot of the hind leg was stimulated by filaments and animal's withdrawal reflex to stimulation was measured by scoring the number of reflexes out of 20 stimuli per each test. The mean value of paw withdrawal frequency for each animal was determined by three independent measurements which were conducted with a 30 min interval between each measurement. We measured paw withdrawal frequency, as an experimental procedure measuring pain responses to mechanical hyperalgesia $[13,14]$.

2.7. DRG Neuron Culture. Rat's sciatic nerves were exposed and subjected to either CI or CCI. DRG at lumbar levels 4 and 5 were dissected and used for primary neuron culture. Neuronal culture was performed as described previously [12]. Dissociated neurons $\left(1.5 \times 10^{2}\right.$ cell $)$ were plated on $12 \mathrm{~mm}$ coverslips in 24-well plate. Cells were cultured for $24 \mathrm{~h}$ and then treated with herbal drugs $(0.5 \mathrm{mg} / \mathrm{ml})$ for $24 \mathrm{hr}$. After fixation, cells were used for immunofluorescence staining as described above.

2.8. Statistical Analysis. Data were presented as mean \pm standard error of mean (SEM). The mean number data among individual groups were compared by the one-way ANOVA followed by Tukey test post-hoc analysis (SPSS computer software version 21.0), and statistically significant differences were reported as $\mathrm{p}<0.05, \mathrm{p}<0.01$, and $\mathrm{p}<0.001$.

\section{Results}

To determine the optimal condition inducing neuropathic pain in rats, we ligated sciatic nerves using two types of sutures and compared pain responses. Behavioral measurement of pain in terms of paw withdrawal frequency revealed that nylon suture was more efficient than silk suture (Figure 1(a)) and thus the nerve ligation in rats was performed using 5-0 nylon thread for the rest of the histobiochemical experiments in the present study. To determine the effects of BGJTD and BeD on behavioral pain responses, we administered BGJTD or BeD for $1-4$ weeks into mice whose sciatic nerves had been ligated for 1 week. Pain susceptibility was not different between BGJTD- and BeD-injected animals after drug administration for 1 or 2 weeks (Figures 1(b) and $1(\mathrm{c})$ ), but the response was significantly improved in animal group administered with $\mathrm{BeD}$ for 3 or 4 weeks compared with CCI+SAL group (Figures 1(d) and 1(e)). Pain susceptibility was also improved by BGJTD treatment but was less effective than $\mathrm{BeD}$ and statistically insignificant compared with CCISAL group.

The abovementioned data indicated that prolonged administration of $\mathrm{BeD}$ was effective for pain regulation. To investigate possible molecular factors related to the regulation of neuropathic pain by $\mathrm{BGJTD}$ and $\mathrm{BeD}$, we prepared rat sciatic nerve which had been subjected to ligation for 1 week following 2 weeks of drug administration and analyzed proteins in the nerve. Levels of BDNF were significantly increased in the nerve after ligation particularly in the proximal portion and remained at similar levels after the treatment of both BGJTD and BeD (Figure 2(a)). BDNF signals were clearly observed in the nerve after ligation (Figure 2(b)), and particularly intense signals were detected around the area of nerve ligation (Figure $2(\mathrm{c})$ ). We also found that TrkB protein, a major neuronal receptor of BDNF, was strongly induced in the nerve after ligation (Figure 2(d)). TrkB level was not altered by BGJTD treatment but significantly decreased in the proximal nerve by $\mathrm{BeD}$ treatment (Figure $2(\mathrm{~d})$ ). TrkB signals were mostly colocalized with NF-200 signals (Figure 2(e)). They were also seen strongly around the area of nerve ligation, which was largely attenuated by treatments of $\mathrm{BeD}$ (Figure 2(f)).

Previous studies have well demonstrated that Erk1/2 protein is activated during the process of nerve repair after peripheral nerve injury [15]. Given that Erk1/2 activity is involved in TrkB signaling in neurons [16], we analyzed phospho-Erk1/2 in an area of nerve ligation and also in DRG neurons at lumbar levels 4 and 5 as retrograde target of sciatic sensory axons. There were some basal levels of phosphoErk1/2 in both the nerve and DRG. Phospho-Erk1/2 was slightly elevated in the nerve after ligation and significantly increased by $\mathrm{BeD}$ (Figure 3(a)). Immunofluorescence staining for the nerve sections from BeD-treated CCI animals showed that while some of phospho-Erk1/2 signals were colocalized with NF-200-stained axons, others were not, suggesting their production from nonneuronal cells (Figure 3(b)). Phospho-Erk1/2 level was significantly increased in the DRG after nerve ligation and further upregulated by $\mathrm{BeD}$ treatment (Figure 3(c)).

To examine whether $\mathrm{BGJTD} / \mathrm{BeD}$ administration affected inflammatory responses in association with neuropathic pain, we analyzed inflammatory cytokines TNF- $\alpha$ and IL-6 in the nerve. TNF- $\alpha$ levels were significantly increased after nerve ligation and then decreased by BGJTD treatment, but not by BeD (Figure 4(a)). Interestingly, however, levels of IL-6 did not show significant changes after CCI and BGJTD treatment but were greatly elevated by $\mathrm{BeD}$ treatment (Figure 4(b)).

It is well known that preconditioning injury (e.g., crush injury on the sciatic nerve) enhances neurite outgrowth of DRG sensory neurons in culture [17]. Here, we investigated the effect of $\mathrm{BGJTD} / \mathrm{BeD}$ on the neurite outgrowth of DRG neurons in rats which had undergone $\mathrm{CI}$ or CCI in the sciatic nerve for 7 days. In DRG neurons prepared after CI, treatments of BGJTD and BeD significantly increased neurite outgrowth (Figure 5(a)). Neurite outgrowth was notably reduced in DRG neurons prepared after CCI, compared with those after CI (see Figures 5(a) and 5(b)). Treatment of BeD, but not BGJTD, significantly enhanced neurite outgrowth (Figure 5(b)). Representative images of DRG neurons are shown in Figure 5(c). Using cultured DRG neurons which were prepared 1 week after nerve ligation, we further analyzed immunofluorescence signals of TrkB protein. TrkB signals were clearly observed in neurons treated with saline vehicle 


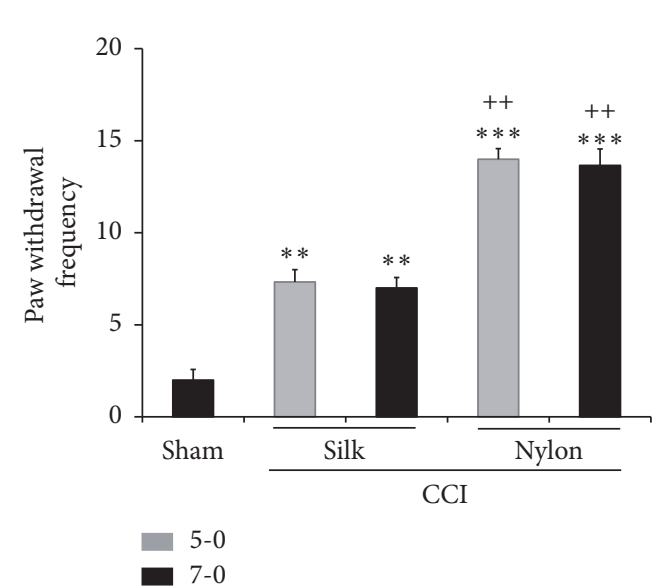

(a)

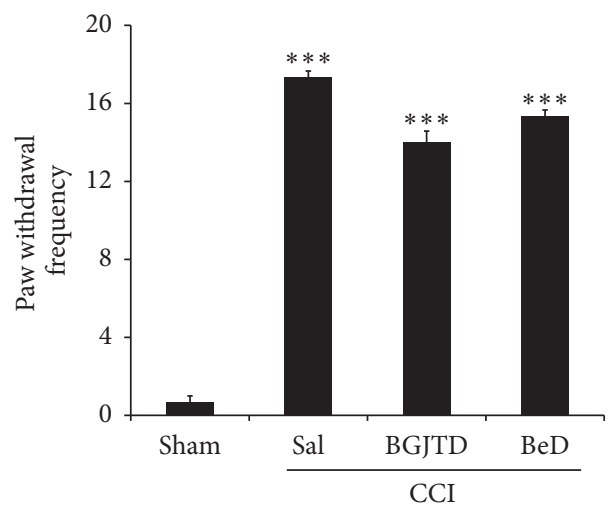

(b)

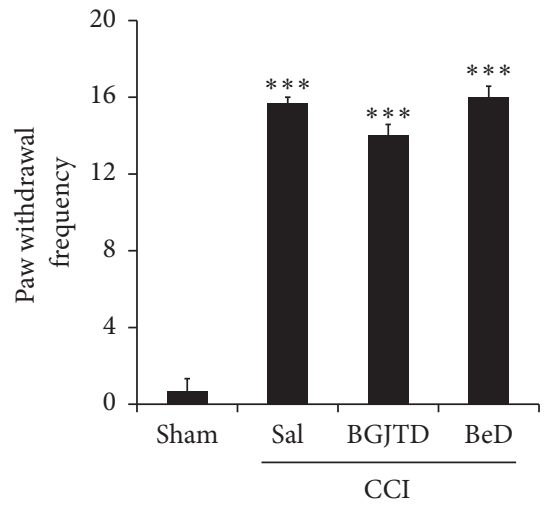

(c)

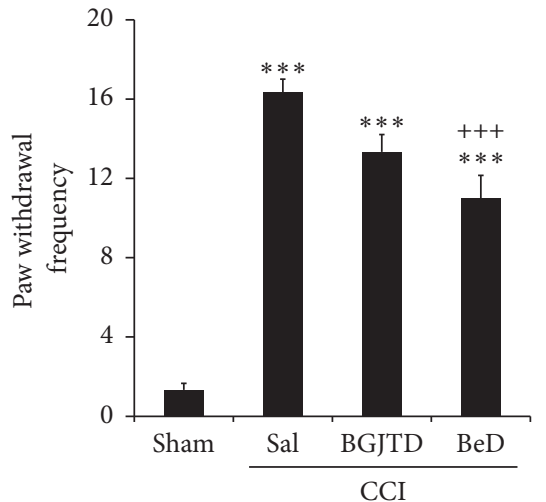

(d)

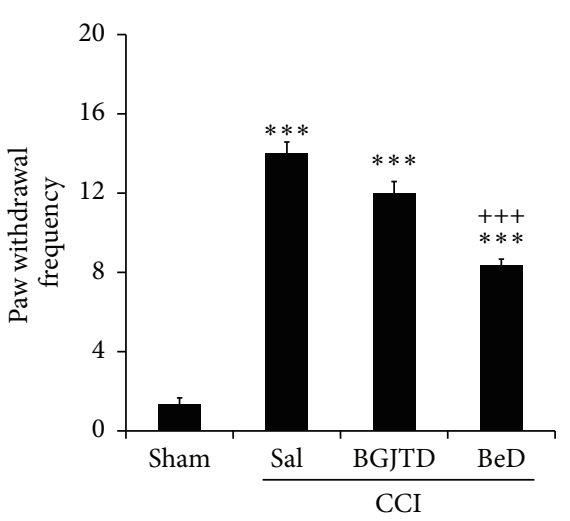

(e)

FIGURE 1: Changes in pain susceptibility in CCI animals by BGJTD and BeD administration. (a) Comparison of pain scores in CCI rats sutured with silk and nylon threads. In each thread, 5-0 and 7-0 sizes were examined. (b-e) Effects of CCI and drug (BGJTD, BeD) administration on pain scores in mice. One week after nerve ligation, animals showing pain scores higher than 15 in terms of paw withdrawal frequency in von Frey test were selected and randomly assigned into different animal groups and administered BGJTD, BeD, and saline (SAL) for 1 week (b), 2 weeks (c), 3 weeks (d), and 4 weeks (e). Number of animals in each group in (a) through (e) $=5$. Error bar $($ mean \pm SEM). $* *$ p $<0.01$ and $* * * \mathrm{p}<0.001$ vs. sham controls. ${ }^{++} \mathrm{p}<0.01$ vs. CCI with corresponding silk threads in (a). ${ }^{++} \mathrm{p}<0.001$ vs. CCI+SAL group in (d, e).

and with BGJTD as well, but the signal intensity was clearly diminished after $\mathrm{BeD}$ treatment (Figure 6(a)). To further examine which herbal constituents were effective in regulating neurite outgrowth and $\operatorname{TrkB}$ expression, we treated cells with individual herbal components of BeD. Neurite outgrowth, as visualized by NF-200-staining of neurons, was prominent in cells treated with PG and PL (Figure 6(b)). Overall TrkB signal intensity in cell groups treated with 4 different herbal constituents was weaker than those of vehicle control group.

\section{Discussion}

In the previous study, we demonstrated that BGJTD and $\mathrm{BeD}$ had a protective effect on neuropathic insults in streptozotocin-induced diabetic animals [11]. Here, using an animal model of CCI, we investigated the effects of BGJTD and $\mathrm{BeD}$ on peripheral neuropathy. BeD administration alleviated pain response in CCI animals and changed the production levels of nerve factors that are related to pathologic responsiveness of pain. BGJTD was partially effective in alleviating pain. $\mathrm{BeD}$ appears to be more effective than BGJTD in regulating the production of TrkB, Erk1/2, and inflammatory cytokine IL-6, raising an issue that it is important to select efficacious components in formulating herbal decoctions while excluding some inefficacious herbal components.

Peripheral nerve ligation is an experimental procedure that is widely used to induce and examine neuropathic pain. We found that pain response in the hind limb was clearly induced after nerve ligation and significantly attenuated by prolonged administration of $\mathrm{BeD}$ lasting 3-4 weeks. We assumed that possible biochemical events, if any, might occur in less than 3 weeks before the behavioral consequences and thus investigated molecular factors that could be involved in pathologic responsiveness in the injured nerve. In the peripheral nerve, BDNF is induced at gene expression levels in neurons and nonneuronal cells such as Schwann cells 


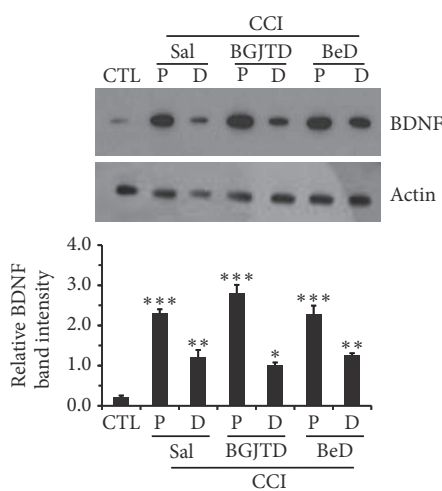

(a)
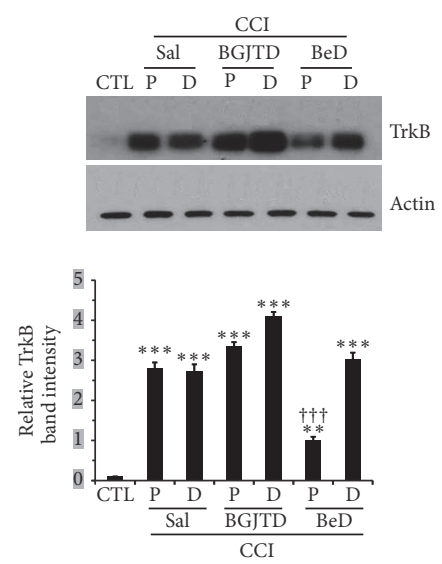

(d)
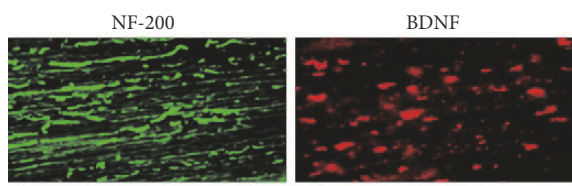

(b)
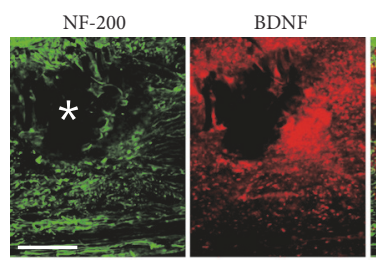

merge

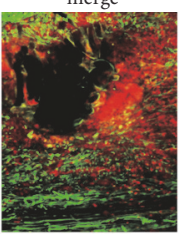

(c)
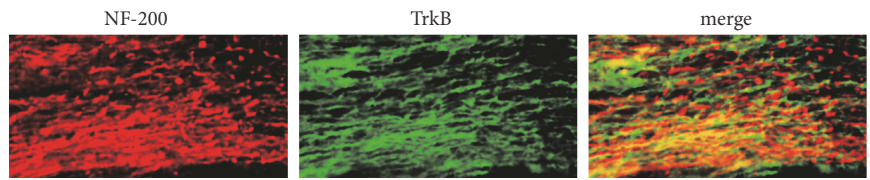

(e)
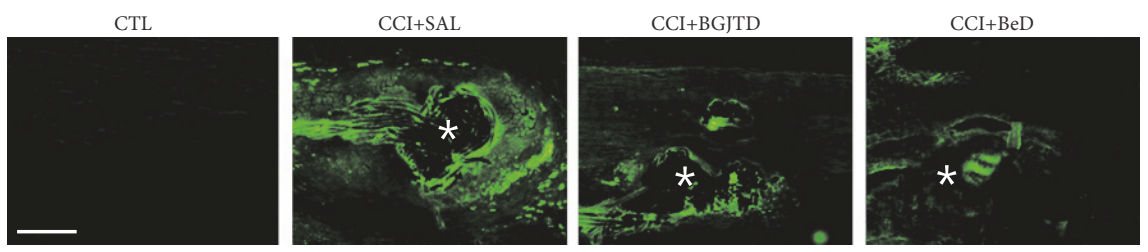

(f)

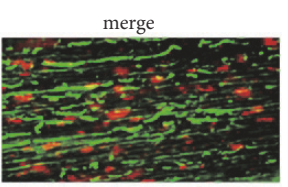
政 


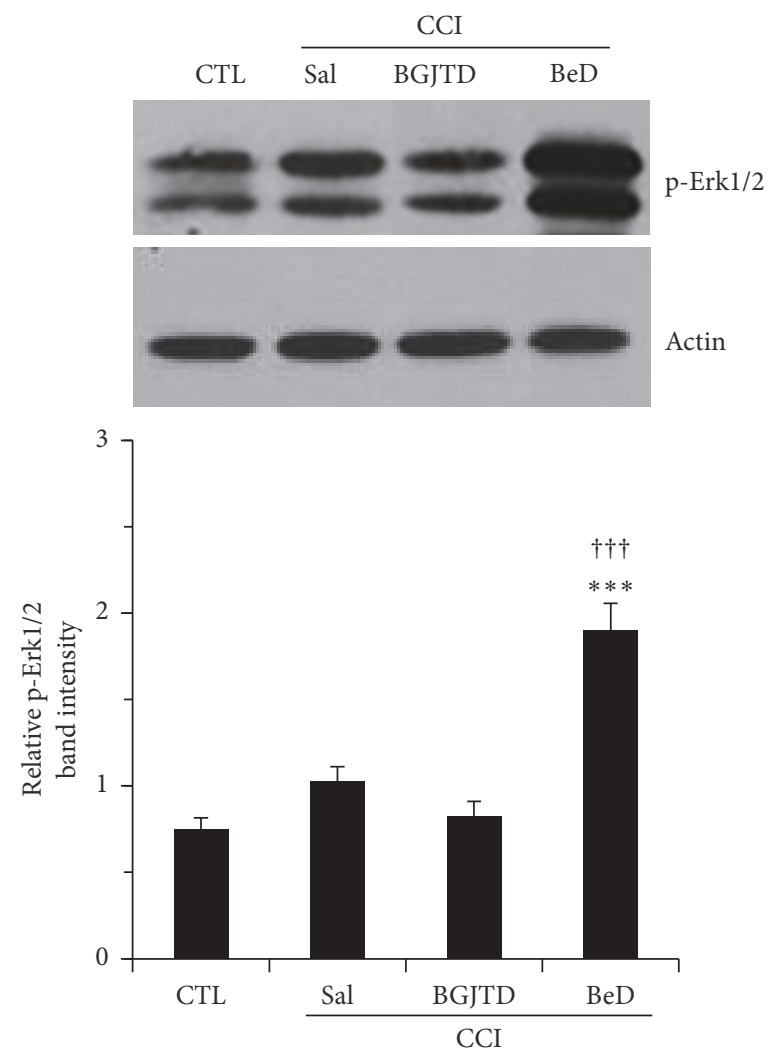

(a)
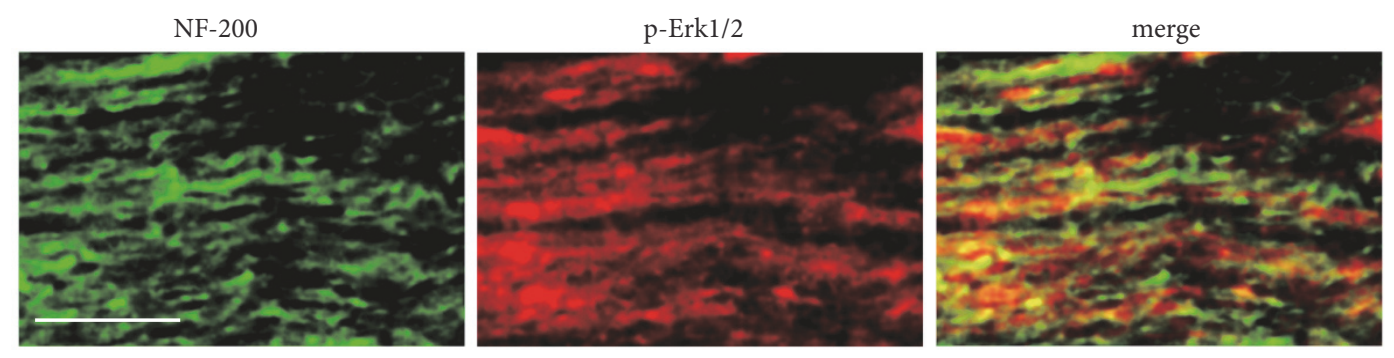

(b)
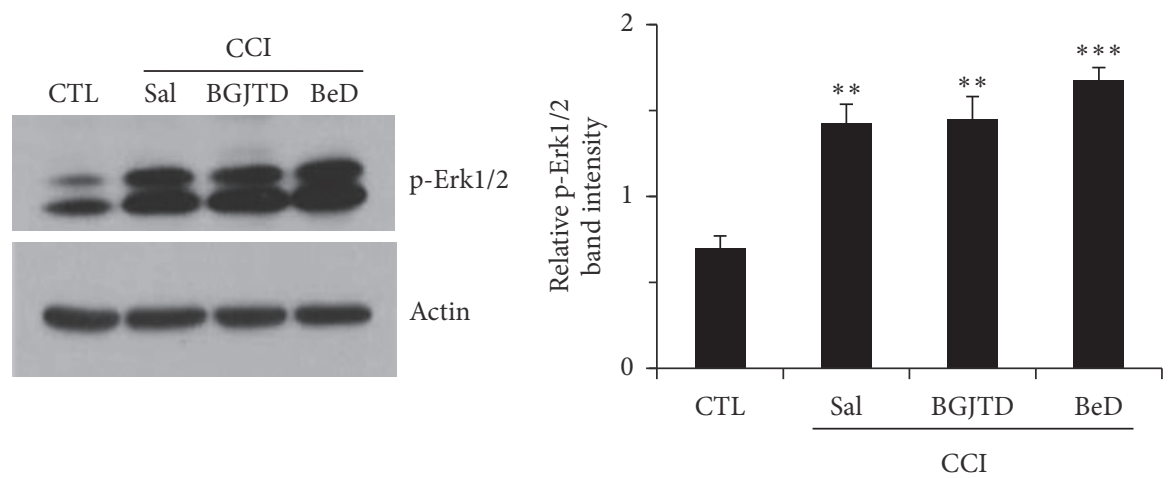

(c)

FIGURE 3: Regulation of Erk1/2 activation in the sciatic nerve and DRG after CCI and BGJTD/BeD treatments. (a, c) Western blot analysis of phospho-Erk1/2 in the sciatic nerve (a) and in the DRG (c). (b) Immunofluorescence images of phospho-Erk1/2 in the sciatic nerve. These are the representative images from an animal treated with CCI+BeD. Western blotting for actin was used as an internal loading control. Bar graphs in (a) and (c) represent quantification of relative band intensity to actin. Error bar (mean $\pm \mathrm{SEM}, \mathrm{n}=4$ ). $* * \mathrm{p}<0.01, * * * \mathrm{p}<0.001 \mathrm{vs}$. control group. ${ }^{+++} \mathrm{p}<0.001$ vs. CCI+SAL group. Scale bar $=100 \mu \mathrm{m}$. 


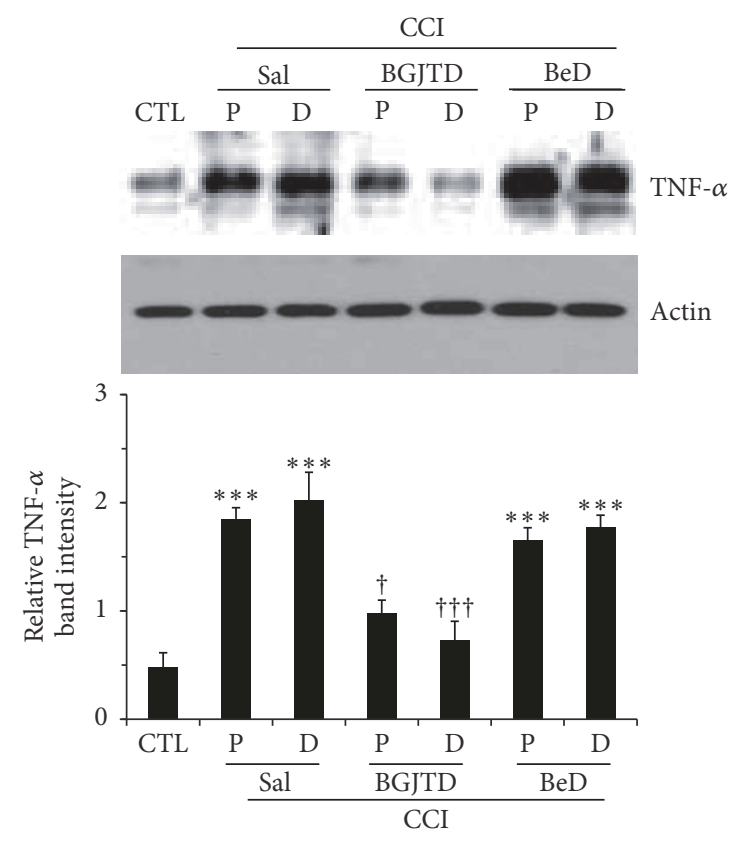

(a)

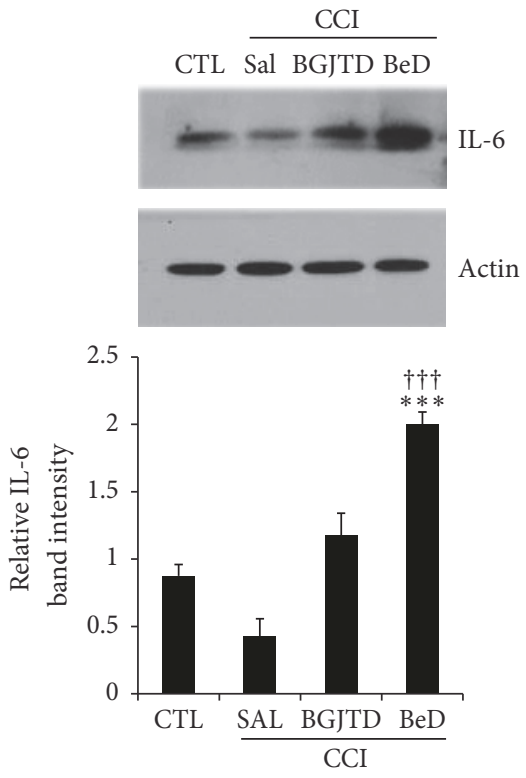

(b)

FIGURE 4: Comparison of TNF- $\alpha$ and IL-6 production in the sciatic nerve after CCI and BGJTD/BeD treatments. Western blot analysis of TNF- $\alpha$ (a) and IL-6 (b) in the proximal (P) and distal (D) stumps of the sciatic nerve. Bar graphs (lower panel in (a) and (b)) represent quantification of relative band intensity to actin. Error bar (mean $\pm \mathrm{SEM}, \mathrm{n}=4) . * * * \mathrm{p}<0.001$ vs. control group. ${ }^{+} \mathrm{p}<0.05,{ }^{+++} \mathrm{p}<0.001$ vs. corresponding CCI+SAL group.

Previous study also demonstrated that Erk1/2 activation is related to an increase of IL-6 synthesis via the activation of NF-kB in astrocytes [28]. IL-6 is known to act as both proinflammatory and anti-inflammatory cytokines [29]. For instance, IL-6 produced from Schwann cells after nerve injury functions to support axonal regeneration and neuroprotection and alleviates neuropathic pain [30,31]. Here, IL-6 level was not increased by CCI, and thus IL- 6 certainly did not act as proinflammatory cytokine at least in the peripheral nerve undergoing neuropathy. Then, $\mathrm{BeD}$ treatment strongly elevated IL-6 level, suggesting the augmentation of antiinflammatory response possibly mediated by IL-6. How would IL-6 produced from Schwann cells and macrophages in $\mathrm{BeD}$-treated CCI animals communicate with injured axons and modulate neuropathic pain? There are IL- 6 receptors in peripheral axons known to transmit signals into JAK/STAT3 signaling molecules in the neuronal cell body $[32,33]$ and induce target gene expression at transcriptional level. TrkB was induced in cultured DRG neurons which were prepared from animals given preconditioning CCI but attenuated by $\mathrm{BeD}$ treatment. We speculate that retrograde signals from IL-6 receptors may be involved in inhibiting TrkB gene expression, which results in reduced TrkB level at the injured nerve area, further leading to a large reduction of pain response (Figure $7(\mathrm{c})$ ). While further study is critical to verify the abovementioned proposal, it seems clear that molecular mechanisms underlying the regulatory effects of BGJTD and $\mathrm{BeD}$ on peripheral neuropathy are different.

Primary DRG neurons can be prepared from adult animals in which preconditioning injury had been given, and their neurite outgrowth is widely used to analyze regenerative responses of injured axons [17, 34]. Using a similar experimental paradigm, we prepared primary DRG neurons after CCI and compared their neurite outgrowth with that of DRG neurons prepared after CI. Neurite outgrowth of neurons from CCI animals was much shorter than those from CI animals. This difference could be partly due to the fact that putative regeneration-inducing signals that might be produced from the distal stump of the injured nerve cannot pass through the constrictive axons. Yet, $\mathrm{BeD}$ treatment promoted neurite outgrowth, implying the potential role of $\mathrm{BeD}$ for the repair process of peripheral axons after ligation injury. When the cells were treated with individual herbal constituents of $\mathrm{BeD}, \mathrm{PG}$ and PL were more effective in enhancing neurite outgrowth than AG and CG. However, all four herbal constituents of BeD similarly attenuated TrkB signals in cultured cells, suggesting that the neuronal responses to individual herbal constituents, in terms of neurite outgrowth and TrkB signaling, were partially, but not completely, correlated to each other. Further study is required to clarify how the intrinsic neuronal ability of axonal regeneration is linked to the regulation of neuropathic pain when $\mathrm{BeD}$ and $\mathrm{BGJTD}$ were administered for therapeutic purpose.

\section{Conclusion}

Our data suggest that BNDF-TrkB signal pathway may be activated within the nerve fibers after CCI and contribute to the transmission of neuropathic pain signals into neuronal cell body. Administration of $\mathrm{BeD}$ may lead to downregulating 


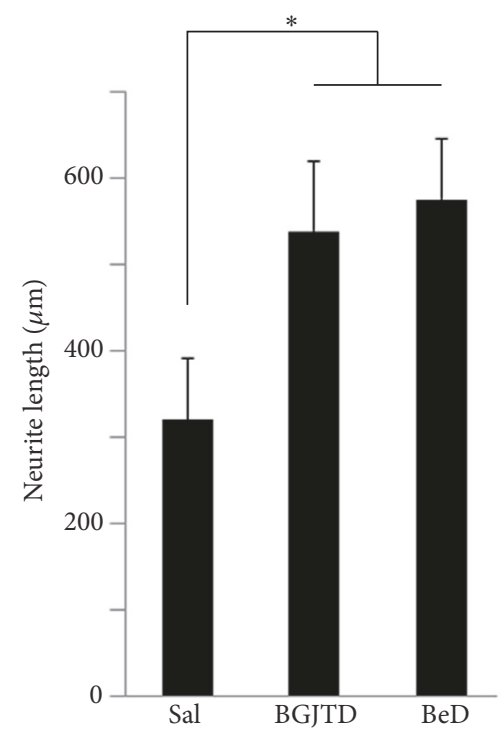

(a)
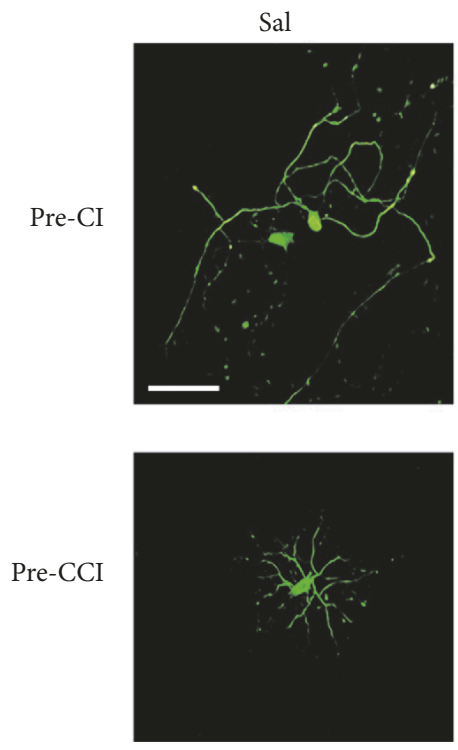

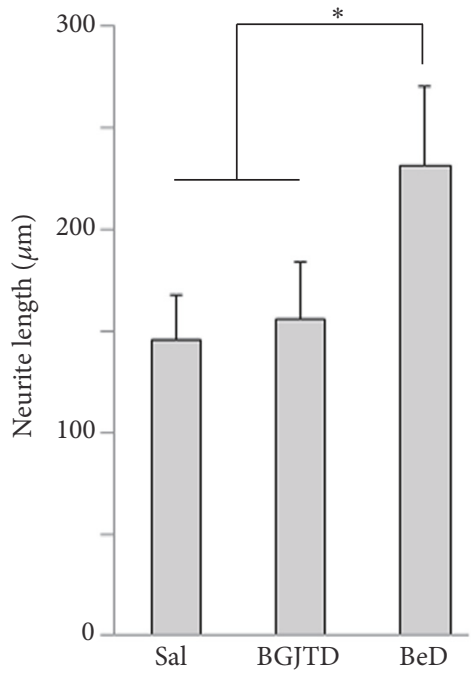

(b)
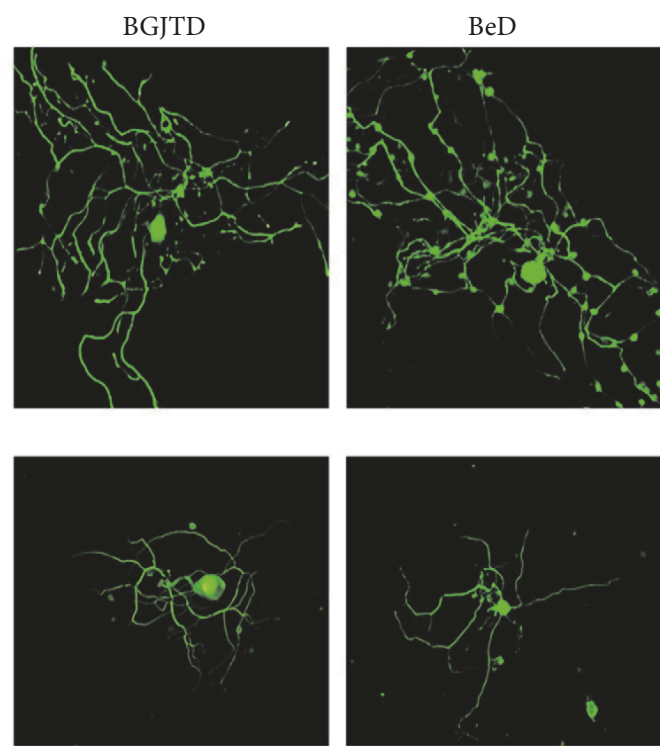

(c)

FIGURE 5: Effects of BGJTD and BeD treatment on the neurite outgrowth in cultured DRG neurons. Primary DRG neurons were prepared from rats which had undergone preconditioning CI (Pre-CI) in the sciatic nerve for 7 days (a) or preconditioning CCI (Pre-CCI) for 7 days (b). (c) Representative immunofluorescence images. Cultured neurons were visualized by green fluorescence of NF-200 protein. Bar plots denote mean \pm SEM $(\mathrm{n}=4) . * \mathrm{p}<0.05$. Scale bar $=100 \mu \mathrm{m}$

TrkB levels in axons and attenuating the transmission of pain signals. Alternatively, BeD may directly act on sensory neurons in DRG or even spinal glial cells and affect pain susceptibility because $\mathrm{BeD}$ was systemically administered [1]. Examination of pain response after focal injection of $\mathrm{BeD}$ into the area of nerve ligation may help clarify this issue. It is unclear at this moment why BGJTD was not effective for the regulation of $\mathrm{BDNF}-\mathrm{TrkB}$ pathway; the effect mediated by negatively acting herbal components that are present only in BGJTD may be considered.

In conclusion, our study highlights that a reconstituted formulation $\mathrm{BeD}$ may be able to produce an effect for the regulation of neuropathic pain comparable with or even superior to the original BGJTD. The experimental approach shown here provides insights into the methodological basis for compositional analysis of multiple herbal constituents of the decoction.
Abbreviations
BGJTD: Bogijetong decoction
BeD: $\quad$ Bogijetong decoction
CCI: Chronic constrictive injury
CI: Crush injury 


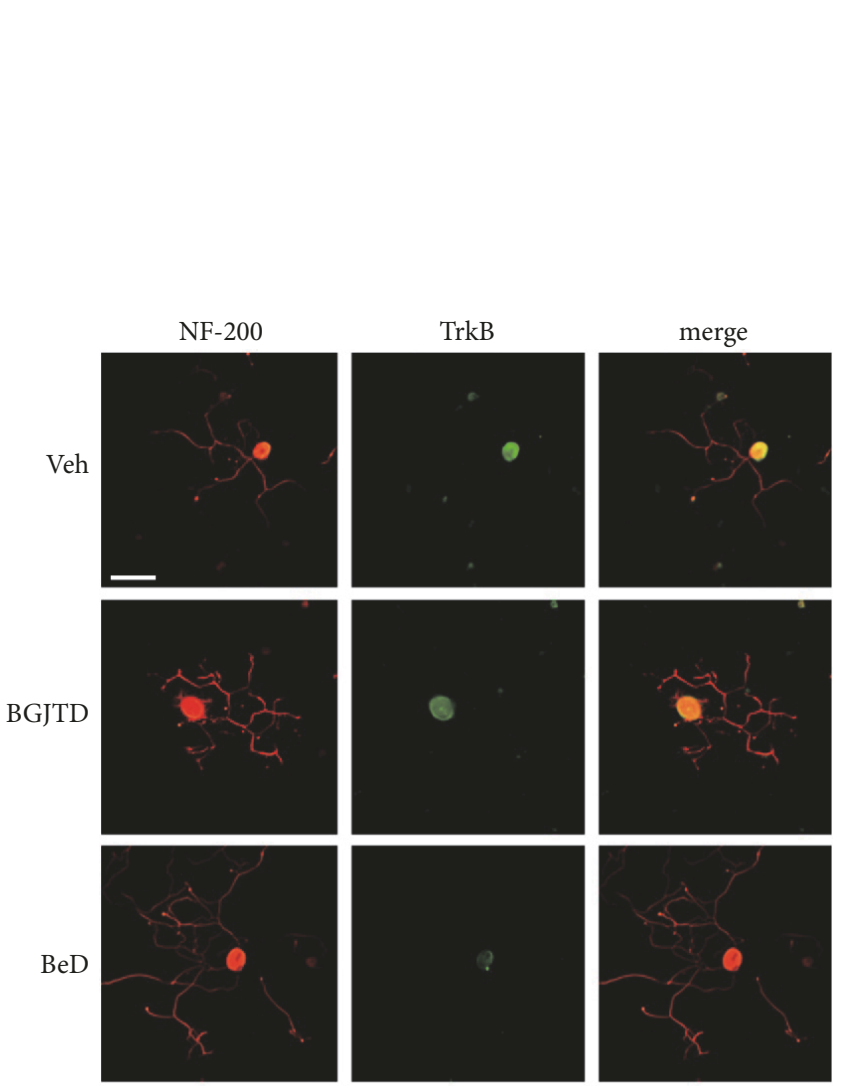

(a)
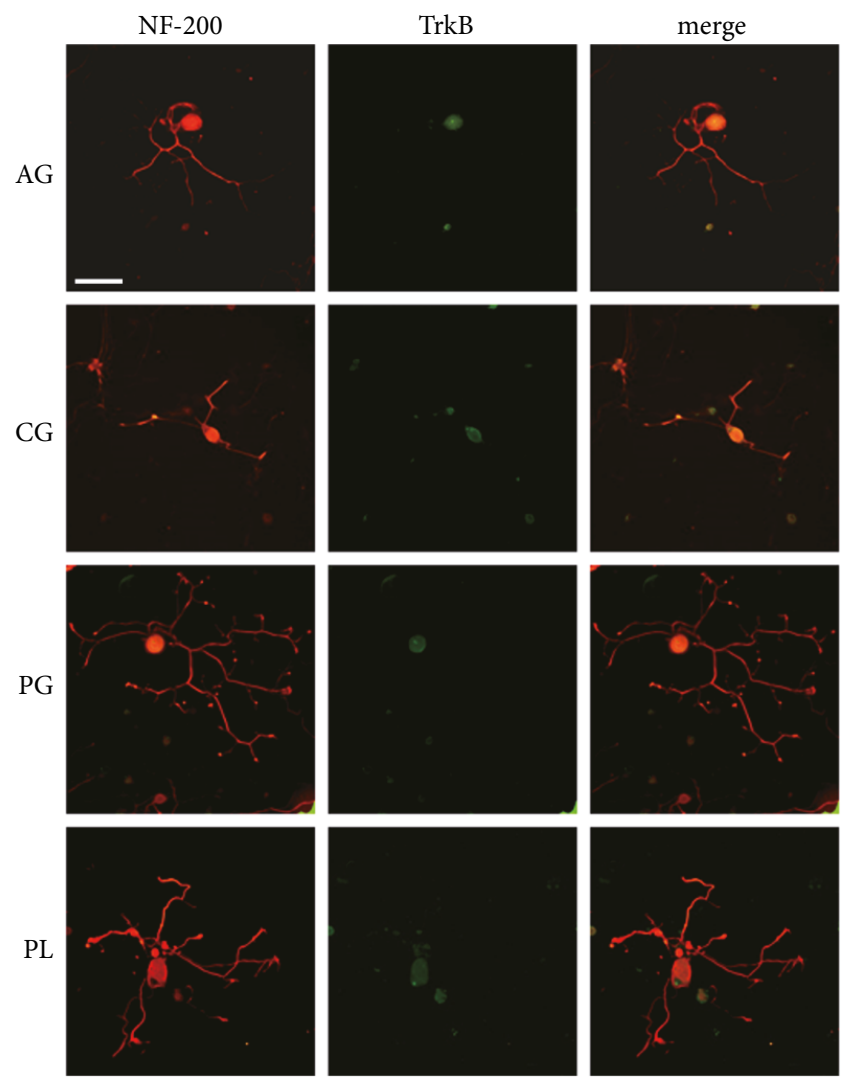

(b)

FIGURE 6: Immunofluorescence analysis of TrkB signals in cultured DRG neurons. Primary DRG neurons were prepared from rats given CCI in the sciatic nerve for 7 days. Neurons were treated with BGJTD and $\mathrm{BeD}$ (a) or individual herbal constituents of $\mathrm{BeD}$ (b) for $24 \mathrm{~h}$ prior to fixation for immunofluorescence staining for NF-200 (red) and TrkB (green) as indicated in the figure. Scale bar $=100 \mu \mathrm{m}$.

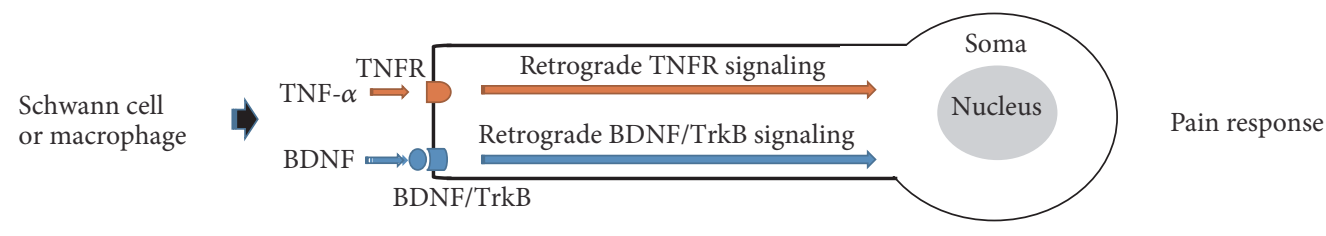

(a)

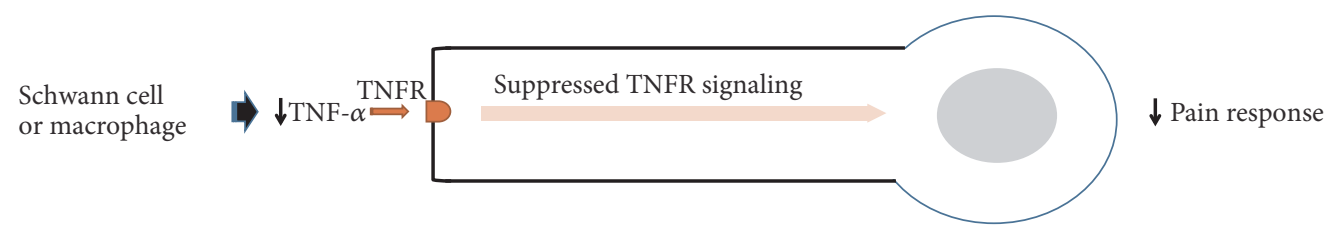

(b)

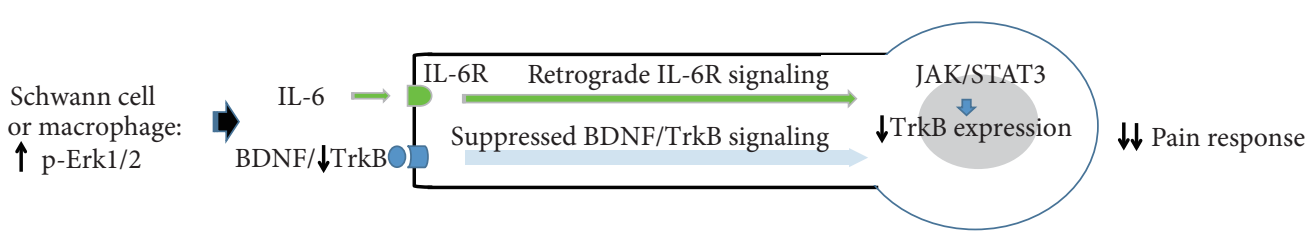

(c)

FIgURE 7: Schematics of proposed mechanisms underlying BGJTD- or BeD-modulated pain responses. According to our data, signaling of TNF- $\alpha$ and TrkB appeared to increase after nerve ligation (a). TNF- $\alpha$, which were decreased by BGJTD treatment, may cause weakening in retrograde signaling of TNFR into the cell body, leading to alleviating pain response (b). After BeD treatment, p-Erk1/2 and IL-6 signals, induced from Schwann cells and macrophages, may transmit the signals of IL-6R into the neuronal cell body (soma), inducing JAK/STAT activation and regulation of target gene expression including the repression of TrkB gene expression (c). Consequently, retrograde TrkB signaling would be attenuated and lead to alleviate pain response. 
DRG: Dorsal root ganglion

BDNF: Brain-derived neurotrophic factor.

\section{Data Availability}

The data used to support the findings of this study are available from the corresponding author upon request.

\section{Conflicts of Interest}

The authors declare no conflicts of interest.

\section{Authors' Contributions}

Uk Namgung and Chung Sik Cho conceived and designed the experiments; Ki-Joong Kim, Hye-Jeong Ahn, Uk Namgung, and Chung Sik Cho performed the experiments, interpreted the data, and wrote the main manuscript; all of the authors read and approved the final manuscript.

\section{Acknowledgments}

This research was supported by the Daejeon University Fund (Grant no. 20160120) (Daejeon, Korea). We thank Dr. Ji-Yeun Park for helpful discussions.

\section{References}

[1] J. Scholz and C. J. Woolf, "The neuropathic pain triad: neurons, immune cells and glia," Nature Neuroscience, vol. 10, no. 11, pp. 1361-1368, 2007.

[2] G. J. Taborsky Jr, B. Ahrén, and P. J. Havel, "Autonomic mediation of glucagon secretion during hypoglycemia: Implications for impaired $\alpha$-cell responses in type 1 diabetes," Diabetes, vol. 47, no. 7, pp. 995-1005, 1998.

[3] N. Khan and M. T. Smith, "Neurotrophins and neuropathic pain: Role in pathobiology," Molecules, vol. 20, no. 6, pp. 1065710688, 2015.

[4] A. K. Clark and M. Malcangio, "Fractalkine/CX3CR1 signaling during neuropathic pain," Frontiers in Cellular Neuroscience, vol. 8, p. 121, 2014.

[5] C. Martucci, A. E. Trovato, B. Costa et al., "The purinergic antagonist PPADS reduces pain related behaviours and interleukin- $1 \beta$, interleukin- 6 , iNOS and nNOS overproduction in central and peripheral nervous system after peripheral neuropathy in mice," PAIN, vol. 137, no. 1, pp. 81-95, 2008.

[6] J. Mika, M. Zychowska, K. Popiolek-Barczyk, E. Rojewska, and B. Przewlocka, "Importance of glial activation in neuropathic pain," European Journal of Pharmacology, vol. 716, no. 1-3, pp. 106-119, 2013.

[7] A. Ledeboer, T. Liu, J. A. Shumilla et al., “The glial modulatory drug AV411 attenuates mechanical allodynia in rat models of neuropathic pain," Neuron Glia Biology, vol. 2, no. 4, pp. 279291, 2006.

[8] J. H. Cho, J. M. Kim, J. H. Kim, Y. S. Oh, and C. J. Kim, "A case report of chemotherapy induced neuropathic pain treated with oriental medicine," The Journal of Korean Oriental Medicine, vol. 31, pp. 58-63, 2010.

[9] J. M. Kim, C. S. Cho, and C. J. Kim, "Clinical study of 8 diabetic patients with paresthesia," Korean Journal of Oriental Internal Medicine, vol. 31, pp. 184-191, 2010.
[10] S. H. Ahn, I. A. Chang, K.-J. Kim, C.-J. Kim, U. Namgung, and C.-S. Cho, "Bogijetong decoction and its active herbal components protect the peripheral nerve from damage caused by taxol or nerve crush," BMC Complementary and Alternative Medicine, vol. 16, no. 1, p. 402, 2016.

[11] K.-J. Kim, U. K. Namgung, and C. S. Cho, "Protective effects of bogijetong decoction and its selected formula on neuropathic insults in streptozotocin-induced diabetic animals," EvidenceBased Complementary and Alternative Medicine, vol. 2017, Article ID 4296318, 12 pages, 2017.

[12] I. A. Chang, M.-J. Oh, M. H. Kim, S.-K. Park, B. G. Kim, and U. Namgung, "Vimentin phosphorylation by Cdc2 in Schwann cell controls axon growth via $\beta 1$-integrin activation," The FASEB Journal, vol. 26, no. 6, pp. 2401-2413, 2012.

[13] N. Goldman, M. Chen, T. Fujita et al., "Adenosine A1 receptors mediate local anti-nociceptive effects of acupuncture," Nature Neuroscience, vol. 13, no. 7, pp. 883-888, 2010.

[14] M. Tsuda, S. Koizumi, A. Kita, Y. Shigemoto, S. Ueno, and K. Inoue, "Mechanical allodynia caused by intraplantar injection of $\mathrm{P} 2 \mathrm{X}$ receptor agonist in rats: involvement of heteromeric $\mathrm{P} 2 \mathrm{X} 2 / 3$ receptor signaling in capsaicin-insensitive primary afferent neurons," The Journal of Neuroscience, vol. 20, no. 15, Article ID RC90, 2000.

[15] T. B. Seo, M.-J. Oh, and B.-G. You, "ERK1/2-mediated schwann cell proliferation in the regenerating sciatic nerve by treadmill training," Journal of Neurotrauma, vol. 26, no. 10, pp. 1733-1744, 2009.

[16] R. A. Segal and M. E. Greenberg, "Intracellular signaling pathways activated by neurotrophic factors," Annual Review of Neuroscience, vol. 19, pp. 463-489, 1996.

[17] D. S. Smith and J. H. P. Skene, "A transcription-dependent switch controls competence of adult neurons for distinct modes of axon growth," The Journal of Neuroscience, vol. 17, no. 2, pp. 646-658, 1997.

[18] M. Richner, M. Ulrichsen, S. L. Elmegaard, R. Dieu, L. T. Pallesen, and C. B. Vaegter, "Peripheral Nerve Injury Modulates Neurotrophin Signaling in the Peripheral and Central Nervous System," Molecular Neurobiology, vol. 50, no. 3, pp. 945-970, 2014.

[19] J. A. M. Coull, S. Beggs, D. Boudreau et al., "BDNF from microglia causes the shift in neuronal anion gradient underlying neuropathic pain," Nature, vol. 438, no. 7070, pp. 1017-1021, 2005.

[20] Y. Yajima, M. Narita, A. Usui et al., "Direct evidence for the involvement of brain-derived neurotrophic factor in the development of a neuropathic pain-like state in mice," Journal of Neurochemistry, vol. 93, no. 3, pp. 584-594, 2005.

[21] S.-J. Geng, F.-F. Liao, W.-H. Dang et al., "Contribution of the spinal cord BDNF to the development of neuropathic pain by activation of the NR2B-containing NMDA receptors in rats with spinal nerve ligation," Experimental Neurology, vol. 222, no. 2, pp. 256-266, 2010.

[22] A. Bhattacharyya, F. L. Watson, T. A. Bradlee, S. L. Pomeroy, C. D. Stiles, and R. A. Segal, "Trk receptors function as rapid retrograde signal carriers in the adult nervous system," The Journal of Neuroscience, vol. 17, no. 18, pp. 7007-7016, 1997.

[23] G. Sabio and R. J. Davis, “TNF and MAP kinase signalling pathways," Seminars in Immunology, vol. 26, no. 3, pp. 237-245, 2014.

[24] V. I. Shubayev and R. R. Myers, "Axonal transport of TNF- $\alpha$ in painful neuropathy: Distribution of ligand tracer and TNF 
receptors," Journal of Neuroimmunology, vol.114, no. 1-2, pp. 4856, 2001.

[25] E. Esposito and S. Cuzzocrea, "Anti-TNF therapy in the injured spinal cord," Trends in Pharmacological Sciences, vol. 32, no. 2, pp. 107-115, 2011.

[26] S. Hanz and M. Fainzilber, "Retrograde signaling in injured nerve-the axon reaction revisited," Journal of Neurochemistry, vol. 99, no. 1, pp. 13-19, 2006.

[27] I. Rishal and M. Fainzilber, "Axon-soma communication in neuronal injury," Nature Reviews Neuroscience, vol. 15, no. 1, pp. 32-42, 2014.

[28] A. Fernandes, A. S. Falcão, R. F. M. Silva, M. A. Brito, and D. Brites, "MAPKs are key players in mediating cytokine release and cell death induced by unconjugated bilirubin in cultured rat cortical astrocytes," European Journal of Neuroscience, vol. 25, no. 4, pp. 1058-1068, 2007.

[29] J. Scheller, A. Chalaris, D. Schmidt-Arras, and S. Rose-John, "The pro- and anti-inflammatory properties of the cytokine interleukin-6," Biochimica et Biophysica Acta (BBA) - Molecular Cell Research, vol. 1813, no. 5, pp. 878-888, 2011.

[30] H. Hirota, H. Kiyama, T. Kishimoto, and T. Taga, "Accelerated nerve regeneration in mice by upregulated expression of interleukin (IL) 6 and IL-6 receptor after trauma," The Journal of Experimental Medicine, vol. 183, no. 6, pp. 2627-2634, 1996.

[31] T. M. Zanjani, M. Sabetkasaei, B. Karimian, F. Labibi, B. Farokhi, and N. Mossafa, "The attenuation of pain behaviour and serum interleukin- 6 concentration by nimesulide in a rat model of neuropathic pain," Scandinavian Journal of Pain, vol. 1, no. 4, pp. 229-234, 2010.

[32] F. Fregnan, L. Muratori, A. R. Simões, M. G. GiacobiniRobecchi, and S. Raimondo, "Role of inflammatory cytokines in peripheral nerve injury," Neural Regeneration Research, vol. 7, no. 29, pp. 2259-2266, 2012.

[33] M. Leibinger, A. Müller, P. Gobrecht, H. Diekmann, A. Andreadaki, and D. Fischer, "Interleukin-6 contributes to CNS axon regeneration upon inflammatory stimulation," Cell Death \& Disease, vol. 4, 2013.

[34] R. Y. Liu and W. D. Snider, "Different signaling pathways mediate regenerative versus developmental sensory axon growth," The Journal of Neuroscience, vol. 21, no. 17, 2001. 


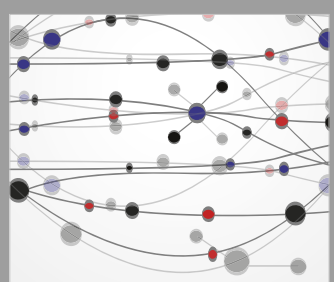

The Scientific World Journal
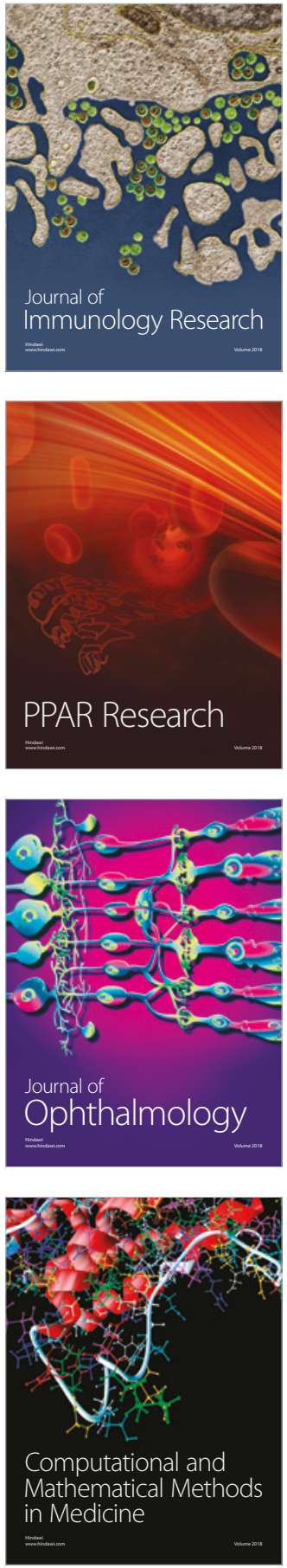

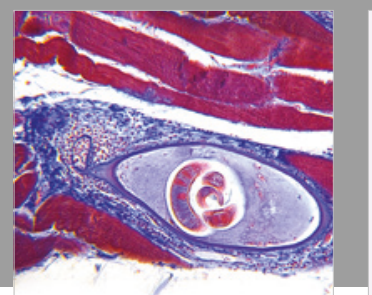

Gastroenterology Research and Practice

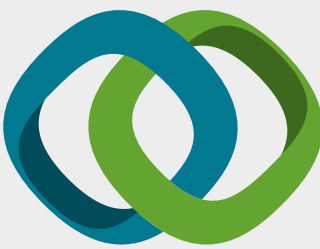

\section{Hindawi}

Submit your manuscripts at

www.hindawi.com
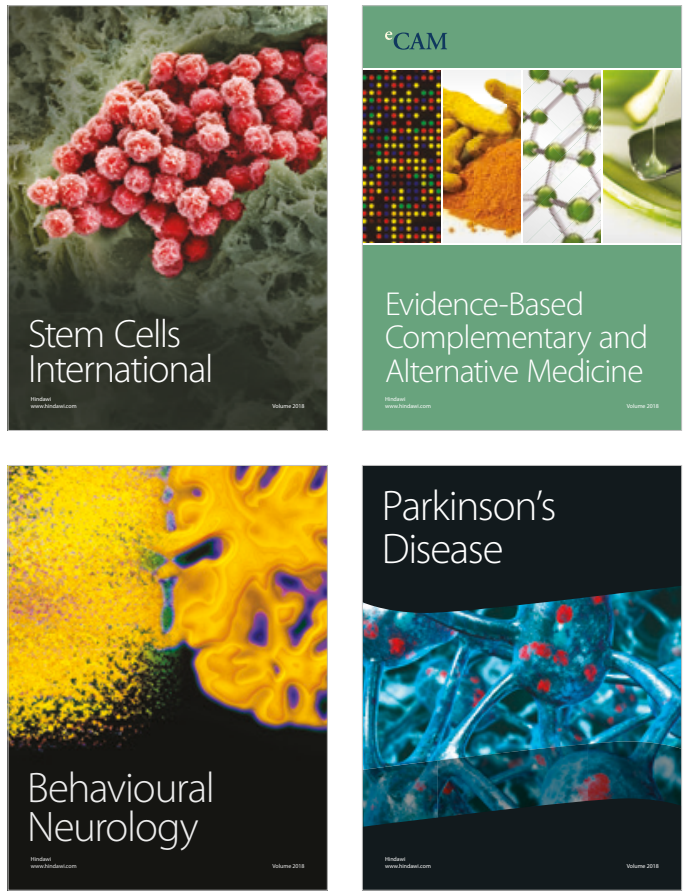

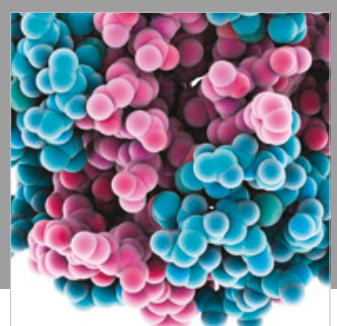

ournal of

Diabetes Research

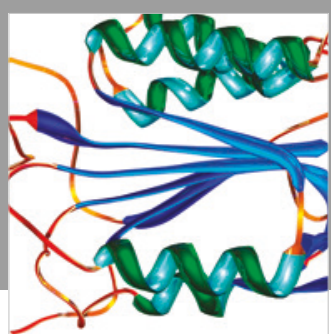

Disease Markers
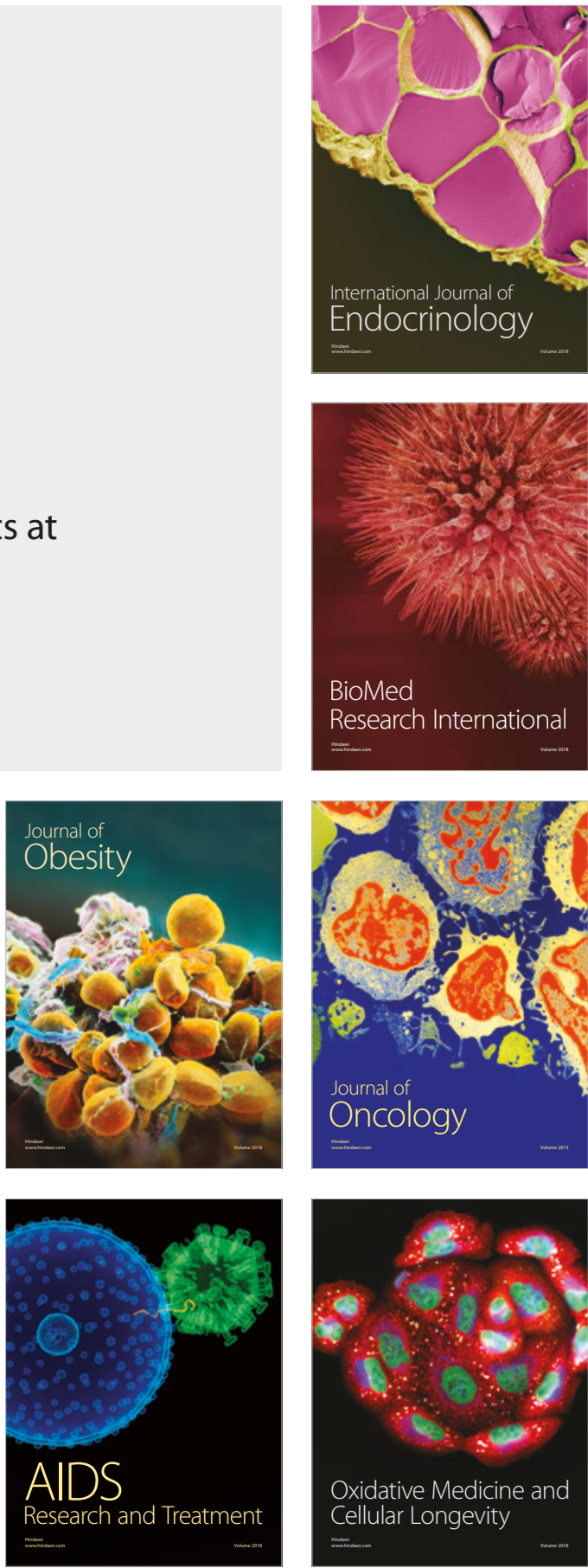